\title{
Digitalisierung im Fremdsprachenunterricht
}

\author{
S. W. Ermolenko*, Y. A. Shcherbonos \\ Staatliche Universität Sumy \\ *Corresponding author. E-mail: s.ermolenko@gf.sumdu.edu.ua
}

Paper received 24.05.20; Accepted for publication 11.06.20.

\section{https://doi.org/10.31174/SEND-HS2020-230VIII38-03}

Abstract. In diesem Artikel geht es um Digitalisierung im Fremdsprachenunterricht, Ansetzen von verschiedenen Tools und Plattformen. Die Digitalisierung eröffnet in vielen Bildungsbereichen neue Chancen und Möglichkeiten. Apps, digitale Klassenzimmer, Chatten aber auch Internetrisiken sind heutzutage Realität geworden. Das Ziel der vorliegenden kurzen Untersuchung ist für uns ein kleiner Versuch die gebräuchlichsten Links, Tools und Werkzeuge im Bereich des Unterrichtes zu präsentieren und ihre Vorteile für den Fremdsprachenunterricht darzustellen. Mit der vorliegenden Arbeit wird ein Anfangsstein für die ausführlichere Studie gelegt.

Schlüsselwörter: Digitalisierung, Fremdsprachenunterricht, digitale Tools, Medieneinsatz, Online-Phase, Medienkompetenz, Motivation

Digitalisierung und digitale Tools umfassen alle Gebiete unseres Lebens und man kann sich heutzutage kaum eine Tätigkeit ohne digitale Einflüsse vorstellen. In der Ukraine existiert seit kurzem das Bundesministerium für Digitalisierung und Wirtschaftsstandort. Man spricht über den Staat in einer App. Man bringt die Digitalisierung voran. Wo es früher Handyverbot stand, sind Handys, Tablets und andere Divises willkommen. Auch in Schuleinrichtungen und noch mehr im Fremdsprachenunterricht. Fast jeder Schüler und Student hat ein Smartphone und Lehrer müssen darauf profitieren und auch diese Gelegenheit in ihren Unterricht einbeziehen. Dabei muss man auch Chancen des digitalisierten Unterrichtes für individuelle Förderung in Betracht ziehen.

Dementsprechend nehmen wir als Gegenstand unserer Untersuchung digitale Tools, Programme und Plattforme. Als Untersuchungsobjekt betrachten wir den Gebrauch und Möglichkeiten der digitalen Tools im Fremdsprachenunterricht.

Die Aktualität unserer Untersuchung sehen wir darin, dass die Digitalisierung die Gegenwart in fast allen Bereichen unserer Existenz geworden ist und dass wir versuchen, einige Links für Fremdsprachenunterricht zu geben und ihre vielseitigen Möglichkeiten vorzulegen, was heutzutage von großem Bedarf ist und von Lehrern in ihrer Arbeit eingesetzt sein kann. Die heutige schwere Situation mit der Quarantäne hat an uns viele neue Herausforderungen gestellt, die auch in der Zukunft unsere Methoden nicht nur in der Präsenz-Phase, sondern auch in der Online-Phase verändern werden.

Die Wissenschaftler, die sich mit diesem Thema auseinandersetzen, bilden die sogenannte Avantgarde und sind Pioniere wie der digitale Deutschlehrer Philippe Wampfler, der bereits sechs Bücher zu diesem Thema publiziert hat. Andreas Pfister als Bildungsjournalist und Philippe Weber als Dozent für Fachdidaktik an der Universität Zürich haben sich auch zum Thema Digitalisierung und Schule mehrmals geäußert. Tobias Zimmermann, der der Digitalisierung eher positive als negative Effekte zu geben scheint. Heike Schaumburg, die in ihrer Studie den aktuellen Forschungsstand zu Chancen und Risiken der Digitalisierung im Unterricht darstellt.

Eine praktische Bedeutung der vorliegenden Arbeit sehen wir in der Tatsache, dass man überall über die Digitalisierung spricht ohne wirklich $\mathrm{zu}$ begreifen, was das bedeutet. Die Herausforderungen der letzten Zeit wie die
Quarantäne in vielen Ländern auch in der Ukraine fordern von uns, den Lehrern, neue Methoden im Fremdsprachenunterricht umzusetzen. Deswegen geben wir eine Reihe von Links, Tools und Plattformen, die Fremdsprachenlehrern ermöglichen, digitale Tools effizienter im Unterricht anzusetzen und sich in diesem digitalen Chaos $\mathrm{zu}$ orientieren.

Das Ziel der vorliegenden kurzen Untersuchung wäre für uns ein kleiner Versuch die gebräuchlichsten Links, Tools und Werkzeuge im Bereich des Unterrichtes zu präsentieren und ihre Vorteile für den Fremdsprachenunterricht darzustellen. Aufgrund der schließenden bzw. geschlossenen Schuleinrichtungen gibt es immer mehr Anfragen, wie man in Zeiten der Corona weiter lernen kann, wie man einen Unterricht digital gestalten kann.

Die Herausforderungen von Heutzutage, das digitale Heute und die noch mehr digitalere Zukunft, so sieht man heute unser Leben. Und für die Schuleinrichtungen wird die Vision des digitalen Lernens großgeschrieben. In der Welt wird immer häufiger über die Digitalisierung in der Bildung, ihre Chancen, Vorteile, Nachteile, Herausforderungen diskutiert. Jedes Land unternimmt seine Schritte um seine Schuleinrichtungen für die digitale Welt vorzubereiten. Man schult die Lehrkräfte aus, damit sie öfter digitale Tools in ihren Unterricht integrieren können. Man sucht nach der perfekten technischen Ausstattung, die sowohl den Lehrern, als auch den Lernenden ermöglichen muss, neue Internetressourcen einzusetzen.

Wenn aber die technische Ausstattung zu wünschen übriglässt, dann muss man schon kreativ sein und nach anderen Wegen suchen, so wie das neue Motto in der Ausbildung „Bring Your Own Device“ (BYOD), indem man das eigene Handy bzw. iPhone im Unterrichtsprozess involviert. Dabei kommt man schon vom Handyverbot in Lehranstalten zu immer häufigeren Mobilgerätegebrauch.

In der Frage der Digitalisierung der Bildung gehen wir in der Richtung, die einige Länder bereits gegangen sind und wir können schon jetzt die Schlussfolgerungen von diesem Prozess betrachten, das für uns wiederum als Vorteil gesehen werden kann. Nach fünf Jahren empirischer Untersuchungen, die vom mmb Institut für einige Institutionen, darunter auch das Ministerium für Bildung, Jugend und Sport des Landes Brandenburg durchgeführt wurden, geben sie Zwischenfazit über die Digitalisierung an Schulen in Deutschland und Bedarfgebiete beim Medi- 
eneinsatz im Unterricht. Darunter sind folgende immer wiederkehrende zu nennen:

1. Passende Ausstattung, die man an vielen Schuleinrichtungen leider noch nicht hat. Dazu gehören in erster Linie WLAN, Beamer, Whiteboards usw.

2. Praxisbezogene Qualifizierung von Lehrern/Lehrerinnen, die heutzutage nicht mehr als Vermittler der Wissen betrachtet werden, sondern als Coaches von verschiedenen Projekten.

3. Pädagogisch-didaktische Beratung und technische Begleitung.

4. Verunsicherung, fehlende Motivation der Lehrkräfte, die Medieneinsatz mehr als Belastung und nicht als Bereicherung betrachten [1].

Man sieht auch die Evolution im Lernen, wo von der Trichter-Methode bis zum Lernen im digitalen Zeitraum gekommen ist, wobei das Unterrichten kreativ, individualisiert, selbstorganisiert, kompetenzorientiert und interdisziplinär sein soll. Man spricht nicht mehr vom Wissen, sondern schon von Kompetenzen und in erster Linie von der entscheidenden Kompetenz die nötige Information $\mathrm{zu}$ finden, zu recherchieren und diese Fähigkeit soll geschult werden. Als Fazit kann man ziehen, dass wichtige Momente auch aus dem Mittelalter geblieben sind, nur zu den neuen digitalen Bedingungen angepasst [2].

Im Jahre 2017 hat die Europäische Kommission den „Europäischen Rahmen für die digitale Kompetenz Lehrender (DigCompEdu) veröffentlicht, der vom GoetheInstitut aus dem Englischen ins Deutsche übersetzt worden ist., wo drei Kompetenzbereiche in digitalen Aspekt unterscheidet werden und zwar

1. Die berufliche Kompetenz der Lehrenden.

2. Die pädagogische Kompetenz der Lehrenden.

3. Die Kompetenzen der Lernenden.

Dabei wird der Selbsteinschätzung der Lehrenden ein großer Wert zugeschrieben, die ihnen ermöglichen soll, ihren eigenen Kompetenzzustand zu verstehen, in welchen Bereichen sie ihre Fähigkeiten und Kompetenzen weiter entwickeln können [3].

Dem oben gesagten entsprechend kann man folgende Definition von der Digitalisierung im Fremdsprachenunterricht geben und zwar als Einsatz der technischen Mittel, der digitalen Medien, die den Lernenden ermöglichen den darzulegenden Unterrichtsstoff besser und anschaulicher darzulegen und beizubringen.

Kommen wir näher zu den digitalen Tools und Online Werkzeugen, die ein Fremdsprachenlehrer im Unterricht anwenden kann. Wir nehmen diese Tools je nach ihrer Funktion und geben einige Tipps, wie man sie im Fremdsprachenunterricht anwenden kann.

Als erstes möchten wir LearningApps präsentieren, die schon mehrere Kollegen benutzen und hier Aufgaben aller Art und ihre Arbeitsblätter ausgearbeitet und heruntergeladen haben. Deswegen kann man hier nach der Registrierung anmelden, zu der Fremdsprache angehen und das gewünschte Thema angeben und abrufen. Man kann diese Arbeitsblätter nicht nur anwenden, sondern auch ähnliche produzieren. Meistens werden hier sowohl grammatische als auch lexikalische Themen behandelt.

Das nächste Werkzeug ist www.quizlet.com, wo man Klassen anlegen, Module vorlegen, den Fortschritt der Lernenden verfolgen, korrigieren und individuelle Aufgaben geben kann. Aber man muss auch berücksichtigen, dass diese Möglichkeit kostenpflichtig ist und nicht alle Schulen bzw. Lehrer sich das leisten können.

Solche Aufgaben wie Quizzes kommen bei Schülern immer sehr gut an und die kann man auf Seiten learningsnacks, www.quizziz.com oder kahoot.it entweder finden oder selbst produzieren, aber hier wird in erster Linie Lexik abgefragt. Diese Aufgabensorte wird in der Form eines Wettbewerbes durchgeführt.

Wortwolken kommen immer im Fremdsprachenunterricht gut an. Dabei wird der Wortschatz abgefragt. Es gibt viele Wortwolken-Generatoren im Internet solche wie wordart.com, wo man nicht nur verschiedenste Wortwolken produzieren, sondern auch die Wortlisten bearbeiten kann, was sehr ansatzwichtig ist, wenn man Wortwolken aus vollständigen Texten erstellen will.

Mind Mapping Online werden immer öfter im Fremdsprachenunterricht angewendet, die man auf www.mind42.com, www.mindmeister.com oder www.bubbl.us ausprobieren und ansetzen kann. Das Erstellen einer Mind Map oder Landkarte der Gedanken macht unsere Ideen anschaulicher und mit mehr Struktur und Übersichtlichkeit, wobei die Bezeichnungen Thema, Oberbegriff (Hauptast, Hauptidee) und Unterbegriff (Zweig, Nebenzweig) erklärt und eingeübt werden.

Die nächste Idee für den Einsatz von digitalen Tools im Unterricht ist der Brainstorming, was aktuell bei der Arbeit in PEP (Praxiserkundungsprojekt) ist. Dabei sind folgende Seiten zu empfehlen wie www.answergarden.ch, www.mentimeter.com und www.tricider.com. Bei dieser Unterrichtsart bekommen die Gedanken und Ideen von Lernenden einen freien Lauf, sie kommen auf solche Weise in Kontakt, reagieren auf die Ideen gegenseitig, sie benutzen ihr Wissen, reagieren spontan. Diese Arbeit eignet sich für die Einführung in das Thema. Dabei wird das Interesse geweckt und neue Ideen und Vorschläge werden produziert, vorgeschlagen und schnell sichtbar und für alle zugänglich gemacht.

Unterricht kann man mit Hilfe von solchen Plattformen wie EduPage, ILIAS, ownCloud, Teamplace, Adobe Connect, itslearning, alfaview, meetzi, Moodle, G-Suite for Education, Cisco WebEx, flipgrid, showbie, Zoom, Socrativ, Google Meet und Google Classroom digital organisieren, indem man nicht nur Unterricht in Form von Konferenzen, die Klassenarbeit mit Schülern die Hausaufgaben für die Schüler, sondern auch Breakout Rooms oder Hangouts Meet für Arbeit in Paaren verwenden kann und wo man auch die Hausaufgaben verteilen, kontrollieren, verbessern und bewerten kann. In der Zeit der Corona werden neue Funktionen von mehreren Plattformen freigeschaltet, indem man Bildschirmpräsentationen, digitale Tafel, Dateien und Lehrmaterialien, Audio und Video, Kollaborative Arbeit in Echtzeit und vieles andere anwenden kann.

Aber das Wichtigste, was wir unseren Schülern, bzw. Studenten beibringen sollen, das ist das Gespräch, die Kommunikation, interaktive Dialogsysteme. Keine andere Methode wird im Fremdsprachenunterricht so oft verwendet wie das Unterrichtsgespräch, wobei man Fragen stellen, verstehen, reagieren und beantworten muss, gemeinsam Meinungen ausdrückt und austauscht. Jeder Fremdsprachenlehrer lernt, wie man solche Gespräche gestaltet und leitet. Und solche Gespräche sind auch mit 
Hilfe von oben genannten Plattformen wie flipgrid, Zoom, Socrativ u.a.m. während der Sessionzeit möglich.

Die Schlussfolgerungen, die man aus der heutigen Situation und der Notwendigkeit der Lehrkräfte online zu unterrichten, gezogen werden müssen sind folgende. Erstens kann sowohl Präsenz- als auch Online- Phasen für das Unterrichten gebraucht und integrativ verzahnt werden. Zweitens fordern digitale Tools und die momentane Notsituation von unseren Lehrkräften mehr Kreativität und methodische Fachkenntnisse, um Unterrichtsziele auf eine andere Stufe zu verschieben. Drittens wird die Medienkompetenz sowohl von Lernenden als auch von den Lehrkräften gefördert. Die Digitalisierung eröffnet in vielen Bereichen viele neue Chancen, auch im Fremdsprachenunterricht. Und der vorliegende Artikel stellt einen Versuch dar, die notwendigen Tools und Internetseiten für einen erfolgreichen Unterricht zu präsentieren.

\section{LITERATURVERZECHNIS}

1. Abgerufen 20. Mai 2020 von https://www.digitalisierung- 3. Abgerufen 24. $\quad$ Mai 2020 von bildung.de/2018/12/07/digitalisierung-in-schule-undunterricht-ein-zwischenfazit/.
2. Abgerufen 23. Mai 2020 von https://www.goethe.de/ins/ua/de/spr/unt/ver/dgk/21609464.ht
ml?fbclid=IwAR2Wj79vzwPAeMKU29Sb0LBpaeq3we3Uq https://www.nzz.ch/meinung/digitalisierung-und-schulepragmatische-antworten-statt-visionen-ld.1430440. TyJ8shtbX17fPis8ZHw4Wn19IY.

\section{Digitalization of foreign language teaching}

\section{S. W. Ermolenko, Y. A. Shcherbonos}

This article is about digitalization of foreign language teaching, using different tools and platforms. Digitalization opens up new opportunities in many areas of education. Apps, digital classrooms, chatting as well as internet risks have become a reality these days. The aim of the present short investigation would be a small attempt for us to present the most common links and tools in the field of teaching and to show their advantages for foreign language teaching. With the present work, a starting stone is laid for the more detailed study.

Keywords: digitalization, language teaching, digital tools, media use, online phase, media competence, motivation. 